\title{
Seminal Vesicle Mixed Epithelial and Stromal Tumor
}

National Cancer Institute

\section{Source}

National Cancer Institute. Seminal Vesicle Mixed Epithelial and Stromal T umor. NCI

Thesaurus. Code C161636.

A rare biphasic neoplasm that arises from the seminal vesicle. It is characterized by the presence of stromal and benign epithelial elements. This category includes cystadenoma, fibroadenoma, and adenosarcoma. 